\title{
Physical vapor deposition synthesis of amorphous silicate layers and nanostructures as cosmic dust analogs
}

\author{
A. De $\mathrm{Sio}^{1}$, L. Tozzetti ${ }^{1}$, Ziyu $\mathrm{Wu}^{2,3}$, A. Marcellii, ${ }^{4,5}$, M. Cestelli Guidi ${ }^{4}$, G. Della Ventura ${ }^{6}$, Haifeng Zhao ${ }^{2}$, \\ Zhiyun Pan ${ }^{3}$, Wenjie $\mathrm{Li}^{3}$, Yong Guan ${ }^{3}$, and E. Pace ${ }^{1,4}$ \\ ${ }^{1}$ Department of Physic and Astronomy, University of Firenze, Largo Enrico Fermi 2, 50125 Firenze, Italy \\ e-mail: pace@arcetri.astro.it \\ 2 Institute of High Energy Physics, Chinese Academy of Sciences, 100049 Beijing, PR China \\ 3 NSRL, University of Science and Technology of China, Hefei, 230029 Anhui, PR China \\ 4 INFN, Laboratori Nazionali di Frascati, 00044 Frascati, Roma, Italy \\ e-mail: marcelli@lnf.infn.it \\ 5 RICMASS, Rome International Center for Materials Science Superstripes, 00185 Rome, Italy \\ ${ }^{6}$ Dipartimento di Scienze, Università Roma Tre, Largo S. Leonardo Murialdo 1, 00146 Roma, Italy
}

Received 20 August 2015 / Accepted 14 February 2016

\begin{abstract}
Cosmic dust grains (CD) are part of the evolution of stars and planetary systems and pervade the interstellar medium. Thus, their spectral signature may be used to deduce the physical features of the observed astronomical objects or to study many physical and chemical processes in the interstellar medium. However, CD samples are available only from sample-and-return space missions. Thus, they are rare and not sufficient to be used to perform laboratory experiments of astrophysical interest, such as to produce reference spectra. In this contribution, we describe a new physical vapor deposition (PVD) technique that allows the production of amorphous samples with controlled chemical and morphological characteristics. In particular, this technique was developed to grow uniform or microstructured layers of $\mathrm{Mg}$-Fe amorphous silicates (olivine or pyroxene) that are materials of wide interest for laboratory experiments. We discuss the first results that were achieved by applying this new synthesis method. The layers were studied by combining infrared spectroscopy, scanning electron microscopy, and X-ray spectroscopy. The X-ray microscopy was used for the first time to characterize the internal structure of the grains in these synthetic samples. Finally, future improvements of the technique and foreseen applications are discussed.
\end{abstract}

Key words. ISM: structure - infrared: ISM - X-rays: ISM - techniques: imaging spectroscopy - instabilities - interplanetary medium

\section{Introduction}

Solid grains are observed in many astronomical objects, such as accretion disks, proto-planetary nebulae, and asteroid and planetoid surfaces (Mizumo et al. 1988; Jaffe et al. 2004). Submicron dust particles in the interstellar medium were also detected by analyzing extinction curves in the visible and ultraviolet spectral ranges (Whittet 2002).

The spectroscopic observation of the interstellar medium gives evidence of the abundance of amorphous dust particles, but does not provide a univocal determination of their composition or quantifies the materials that are present (Pollack et al. 1994; Draine 2011). The composition of solid grains is inferred by depletion observations, that is, by measuring the lack of heavy elements in the gas phase with respect to those expected assuming solar abundance (Draine 2011). Crystalline cosmic dust grains (CD) were observed by the ISO satellite in circumstellar dust, comets, or in dense structures like accretion disks (Crovisier et al. 1999, 1997; Malfait et al. 1998). The ratio between the amount of crystalline phases and the amorphous phase depends on the evolution of the CD. In particular, specific environmental conditions of the host - such as temperature, photons and particle irradiation, and disk density - that are different from those of the interstellar medium allow the rearrangement of the grain structure to produce long-range orderings
(Pollack et al. 1994; Draine 2011; Colangeli et al. 2003; Gail \& Tscharnuter 2007). Hence, the correct interpretation of the spectroscopic features of both the amorphous and crystalline CDs is crucial for determining their formation and evolution, and consequently, the local physical parameters of astronomical objects not achievable in other ways. The interaction of CDs with the surrounding environment and its role in the local chemistry evolution is another important question (Draine 2011; Colangeli et al. 2003; Jing et al. 2011, 2012). Therefore, laboratory experiments have to provide clues that link the spectroscopic information to the parameters depending on in situ conditions. Unfortunately, the availability of CD samples is extremely limited, and terrestrial silicates have not exactly the same features in terms of morphology and/or composition (Colangeli et al. 2003; Apai \& Lauretta 2010). Synthesis and analysis techniques of cosmic dust analogues (CDA) must therefore be arranged to obtain sufficient samples for laboratory experiments or to provide a detailed cross-characterization of materials (morphological, chemical, and/or structural features) and their spectroscopic signature. Synthetic materials should reproduce closely specific or generally relevant features of natural CDs, so that the chemical, morphological, and structural variations in the CDA or in the surrounding environment during the experiments are representative of what is expected in the interstellar medium or in the circumstellar environments. The production and characterization 
of submicron mineral particles plays a fundamental role in many astrophysical researches (Draine 2011; Colangeli et al. 2003; Apai \& Lauretta 2010).

Several techniques to synthesize cosmic dust analogs were studied (Colangeli et al. 2003). Some of these make use of powdered natural minerals and rocks as starting materials. The advantage of these techniques is that large amounts of dust particles are produced, but their characteristics in terms of morphology, lattice stress, crystallization state, grain size, etc. cannot be a priori controlled. This condition may affect their spectroscopic signatures (Henning \& Mutschke 2010). To produce submicrometric particles equivalent to natural CDs, condensation processes from dense plasma were also tested. These techniques use a natural mineral or a stoichiometric oxide mixture as a starting material. The sample is then vaporized at low pressure with a high oxygen partial pressure to obtain dense plasma and fast oxidization of all atomic components. To avoid any distillation process of the sample, a high-energy density must be dissipated; otherwise, separate evaporation of the different atomic species is induced as a result of their different vapour pressures. A highenergy pulsed laser (PLD; Colangeli et al. 2003) or an arc discharge (Rotundi et al. 2000) is commonly used as heating source. The oxidization of the plasma and the consequent condensation processes in a low-vacuum chamber allows the synthesis of particles with characteristics close to natural CDs (Rotundi et al. 2002). On the other hand, the production rate using these techniques is very low, and a long time is required to collect a tiny amount of these materials.

The present work describes the first results obtained with a novel technique to synthesize glassy (amorphous) layers and CD nanoparticles with the stoichiometric composition of silicates. This method makes use of the well-established physical vapor deposition (PVD; Mann 1964; Mahan 2000; Westwood 1989) thin-film technology modified to condensate amorphous layers with specific composition. Furthermore, using the seeded substrates developed on purpose allows the production of microstructured surfaces with a roughness and morphology similar to CDs. This growth process is carried out in low-pressure controlled atmospheres that are chemically active during the deposition procedure.

The condensation process from the PVD source is uniform onto semispherical surfaces centered on the PVD source itself. Thus, this process allows obtaining extended thin material layers whose extension depends on the distance from the source, while the amounts of material depends on the thickness of the condensed layer.

At ambient or lower temperature, the substrate composition has no influence on the chemical structure of the condensed film, thus selected materials can be used for specific purposes: a plastic flexible material can be used to produce free-standing films, or optical/IR grade windows can be used to perform transmission measurements. Moreover, specific substrates can be coated with synthetic silicates for chemical experiments because it is possible to know and control the exact surface area involved in the experiment.

It must be noted that this deposition technique was developed to produce materials suitable for experimental astrophysics and not to reproduce a specific astrophysical process. It is well known from previous studies (Draine 2011) that all the carbon atoms are chemically bound in carbon monoxide (CO) configurations in systems where the $\mathrm{C} / \mathrm{O}$ ratio is $<1.0$ and the CDs are mainly composed of silicates. Silicates are more abundant than other minerals in many astronomical environments such as star formation regions or accretion disks
(Pollack et al. 1994; Sandford 1996). For this reason, our first experiments were focused on the production of silicate particles, in particular on those phases containing magnesium $(\mathrm{Mg})$ and iron $(\mathrm{Fe})$, like olivine and pyroxene, which are by far the most common silicates in extraterrestrial materials (Draine 2011; Min et al. 2007; Greenberg \& Li 1996; Verhoelst et al. 2009). In addition, taking into account the standard parameters of the process that we discuss below, such as temperature (from $\sim 1200 \mathrm{~K}$ to $\sim 2400 \mathrm{~K})$ and pressure $\left(10^{-3}\right.$ mbar), we can infer that a synthesis like this can occur in hot Jupiter-like exoplanets. It was previously argued that silicate clouds are present in this class of exoplanets (Demory et al. 2013).

Near-infrared (NIR) spectroscopy, scanning electron microscopy (SEM), X-ray microscopy, and energy dispersive X-ray spectroscopy (EDX) techniques were used to characterize the mineralogy, the chemical composition, the morphology and the internal structure of the synthesized CDs.

\section{Experimental procedures}

\subsection{Starting materials}

Natural olivine is commercially available; thus it was selected as starting target material. Olivines are solid solutions between iron and magnesium end members described by the ideal chemical formula $\left(\mathrm{Mg}_{x} \mathrm{Fe}_{1-x}\right)_{2} \mathrm{SiO}_{4}$, where $0<x<1$ indicates the fraction of $\mathrm{Mg}$ (atoms per formula unit, apfu) in the silicate. The systems whose stoichiometry corresponds to $x=1$ and $x=0$ are named forsterite and fayalite, respectively. ( $\mathrm{Mg}, \mathrm{Fe})$-pyroxenes have the ideal chemical formula $\left(\mathrm{Mg}_{x} \mathrm{Fe}_{1-x}\right) \mathrm{SiO}_{3}$, where for $x=1$ and $x=0$ the end-members are enstatite and ferrosilite, respectively. It is worth stressing that $\mathrm{Ca}$-free pyroxenes in the $\mathrm{MgO}-\mathrm{FeO}-\mathrm{SiO}_{2}$ system have orthorombic symmetry, whereas Ca-bearing pyroxenes are structurally monoclinic (Deer et al. 1997).

\subsection{Evaporation source and the substrates}

The heating source was a standard electron beam (EB) focused on the material to be evaporated. This heating source was selected for its potential capacity to quickly reach temperatures of up to $10000 \mathrm{~K}$, allowing the complete melting and the following fast evaporation of all the materials in the crucible.

The final temperature of the starting material during the deposition depends on its characteristics because the temperature does not change during the transition between two physical states (liquid and gas in this case). As a result of the high forsterite content of the starting material, its melting point is around $2163 \mathrm{~K}$ and the vapor temperature around $2250 \mathrm{~K}$ and $2500 \mathrm{~K}$. This latter value strongly depends on the pressure of the deposition chamber and on the iron content in the starting mineral.

In this device, the EB produced by thermoionic emission by a tungsten filament is accelerated up to $8 \mathrm{kV}$ and bent by a controllable magnetic field to impinge and thermalize onto the target. To increase the heating uniformity and the amount of evaporating material, the EB can be swept by varying the intensity of the bending magnetic field. Both the heating and deposition rate can be adjusted by tuning the intensity of the thermoionic emission. The energy of the EB, dissipated onto the small sample surface, induces complete melting and vaporization of the target material. The vapor cloud expands hemispherically from the source until a condensation onto a cold substrate occurs. Since the cloud 
Table 1. Relative elemental abundance in the natural olivine used in our evaporation process and in the deposited layers as measured by EDX analysis.

\begin{tabular}{lcc}
\hline \hline & Natural olivine & Evaporated material \\
\hline $\mathrm{MgO}$ & $49 \%$ & $45 \%$ \\
$\mathrm{FeO}$ & $7 \%$ & $6 \%$ \\
$\mathrm{SiO}_{2}$ & $44 \%$ & $47 \%$ \\
\hline
\end{tabular}

is intrinsically homogeneous, the uniformity of the thickness of the deposited film only depends on the distance from the source and on the solid angle of the cloud that is intercepted by the substrate. Thus, standard PVD techniques can be used to produce thin uniform films of the evaporated material.

As already mentioned, the chemical composition and the structure of the substrate in our experiment do not influence the final chemical structure of the condensed film. Thus, specific substrates were selected for our purposes. Temperatureand vacuum-compatible polyamide Kapton films were used as substrate, for instance, enabling the following removal of the condensed material to avoid the substrate affecting the experiments and the analysis. In principle, any substrate stable under thermal and vacuum conditions used during the evaporation and condensation process can be used. Materials that are transparent in the optical or IR range, for example, can be selected for further spectroscopic analyses in transmission mode or, alternatively, substrates developed on purpose to be used in other types of experiments.

\section{Results}

\subsection{Evaporation materials and the active atmospheres}

The EB PVD deposition technique allows both minerals and oxide mixtures as starting evaporation material. The oxygen fugacity, that is, the $\mathrm{O}_{2}$ partial pressure, $P_{\mathrm{O}_{2}}$, has to be controlled during the evaporation because a loss of $\mathrm{O}_{2}$ from the system may have a significant effect on the final stoichiometry, that is, on the cation/oxygen ratio and the aggregation state of the deposited material. It is possible to control the oxygen content of the deposited material by modulating the $P_{\mathrm{O}_{2}}$ during the deposition process. This technique, called reactive deposition, uses a starting weak gas flux to purge the deposition chamber; then, by controlling the partial pressure of one or more gases, in our case $\mathrm{O}$, and the deposition rate, the actual composition of the vapor phase can be tuned. In the experiment described here, an oxygen-rich atmosphere was introduced into the deposition chamber to compensate for any $\mathrm{O}_{2}$ loss.

To obtain a deposited layer with characteristics similar to those of silicate CDs, a natural olivine with $x \sim 0.9$, close to the composition of forsterite, was used as starting material. The olivine was preliminarily characterized with EDX and FTIR spectroscopy to be compared later with the spectra of the deposited layers. These preliminary depositions were uniform, covering flat substrates, with thicknesses ranging between $0.5 \mu \mathrm{m}$ and $10 \mu \mathrm{m}$ and with an accuracy better than $50 \mathrm{~nm}$. The iron oxidation in the deposited material was characterized by XAS spectroscopy at the Fe K-edge at the B18 beam line of the diamond light source at Oxford. Analysis of the pre-edge structure ruled out, within experimental errors, the presence of $\mathrm{Fe}^{3+}$ in the examined sample, thus confirming that the expected stoichiometry was achieved (Wu et al. 2004). The deposited layers were removed from the substrate to be studied. Table 1 gives

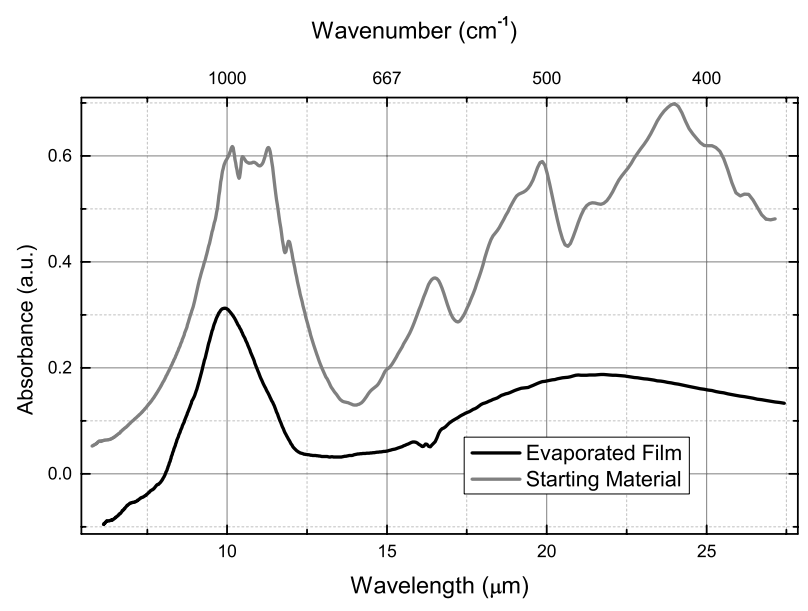

Fig. 1. Absorbance FTIR spectra of a natural olivine used as starting material (top) and of the evaporated and condensed layer (bottom). The peaks observed in the top spectrum can be assigned to the $\mathrm{Si}-\mathrm{O}$ stretching modes of a crystalline olivine. The broad absorption of the evaporated sample is compatible with a glassy state of the synthesized material.

the relative oxide abundance obtained by EDX measurements. The first column shows the values for the natural starting material, the second the values for the deposited layers. Small differences $(\sim 1 \%)$ were detected within the single olivine crystal, although large data scattering was observed among different crystals $(\sim 7 \%)$.

An excellent homogeneity of the oxide ratio was observed in the evaporated material in the same deposition run $(\sim 1 \%)$, but larger differences (up to 5\%) were observed for different crystal mixtures in the evaporation crucible. Therefore, data reported in Table 1 are averaged on several deposition runs.

Representative fragments of both natural olivine and evaporated materials were analyzed by Fourier transform infrared spectroscopy (FTIR) in the $2.5-25 \mu \mathrm{m}$ spectral range. Samples were prepared as pellets, mixing approximately $0.5 \mathrm{mg}$ powder with $150 \mathrm{mg}$ of $\mathrm{KBr}$. Data were collected at the SINBAD beamline at $D A \Phi N E$ (INFN, Laboratori Nazionali di Frascati) in transmission mode, using a Globar source, a $\mathrm{KBr}$ beam splitter, and a DTGS detector, with a nominal resolution of $4 \mathrm{~cm}^{-1}$. We averaged 128 scans for both sample and background (Cestelli Guidi et al. 2005). We compare in Fig. 1 the FTIR spectrum of the evaporated material (bottom) with that of the natural olivine used as source material.

The olivine spectrum (top) is characterized by a group of overlapping but resolved peaks between 9 and $12 \mu \mathrm{m}$ and by additional absorptions at wavelengths $<15 \mu \mathrm{m}$. The former can be assigned to the stretching modes of $\mathrm{Si}-\mathrm{O}$ bonds of the $(\mathrm{Mg}, \mathrm{Fe})_{2} \mathrm{SiO}_{4}$ compound in a crystalline state (Colangeli et al. 2003; Della Ventura et al. 2014). The multiplicity of the bands in the $9-12 \mu \mathrm{m}$ range suggests the distorted symmetry of the $\mathrm{SiO}_{4}$ tetrahedron in the olivine structure (Duke \& Stephens 1964). The other peaks can be assigned to the $\mathrm{Si}-\mathrm{O}$ bending modes $\left(16\right.$ and $20 \mu \mathrm{m}$ ) and possibly to the $\mathrm{M}-\mathrm{O}$ (with $\mathrm{M}=\mathrm{Mg}, \mathrm{Fe}^{2+}$ ) stretching modes $(<20 \mu \mathrm{m})$. These spectra of crystalline olivine were observed in astronomical environments before (Molster et al. 1999, 2002; Bouwman et al. 2003). It is known from the mineralogical literature that the frequencies of the bands observed in the olivine spectra are a function of the $\mathrm{Mg} / \mathrm{Fe}^{2+}$ composition of the phase (Duke \& Stephens 1964). In particular, the higher frequency component close to $10 \mu \mathrm{m}$ is typical for an almost end-member forsterite, in agreement with the chemical 


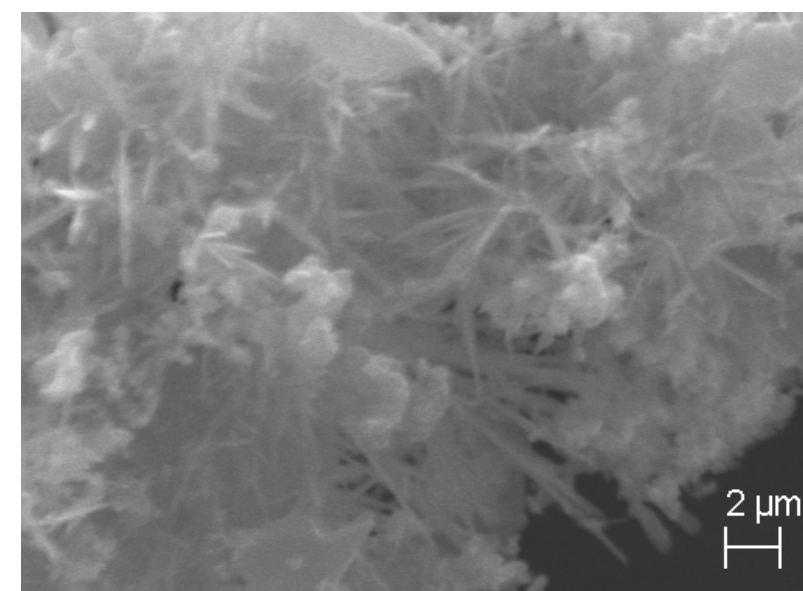

Fig. 2. SEM image of the iron oxide seeds.

composition given above. The frequencies of the $<15 \mu \mathrm{m}$ bands, see for example the position of the $16 \mu \mathrm{m}$ peak, are also indicative of an almost $\mathrm{Mg}$-pure olivine.

The IR spectrum of the evaporated material in Fig. 1 shows two very broad and convoluted main absorption features centered at $\sim 10$ and $22 \mu \mathrm{m}$. Such broad bands are typical of amorphous silicates and were previously reported as reference absorptions for olivine crystallization experiments (Murata et al. 2007). Therefore, combining the information from the EDX and FTIR, we can conclude that our PVD process allowed the synthesis of silicate glassy films with a composition similar to that of the starting material. This is indeed a first notable result because it provides evidence that our technique allows the reproduction of a wide class of amorphous silicates that are potentially present in the interstellar medium. Indeed, some of the homogeneous layers we produced with our technique were previously used by Jing et al. $(2011,2012)$ to investigate the formation of water and molecular oxygen in the interstellar medium (Jing et al. 2011, 2012). Moreover, the capability of synthesizing amorphous silicates with controlled physical and chemical properties can also provide suitable starting materials for crystallization experiments under variable environmental conditions, for instance, increasing temperature or pressure.

\subsection{Substrate seeding}

Many laboratory experiments in astrophysics and astrobiology require $\mathrm{CDs}$ with roughness or porosity equivalent to those of agglomerated powders or simulating the surface of an asteroid. To produce particles and/or nano-structured surfaces with our technique, a specific substrate treatment was developed in which the particles on the substrate act as seeds for the growth of nanosized structures. Of the possible nano-seed materials, iron oxides were selected because of their morphological features and because $\mathrm{Fe}$ and $\mathrm{O}$ are components of the evaporated olivine. The seeds were obtained by degrading a commercial pure iron powder with deionized water. This material was treated and dispersed on the substrate surface to achieve uniform distribution of seeds.

The structure of each seed is extremely complex and composed of several hundred bushes consisting of needle-shaped nanometric crystals (Fig. 2). The seeds are dispersed on the substrate in such a way as to obtain a uniform distribution and avoid large clusters. Particles and nano-structured surfaces were then

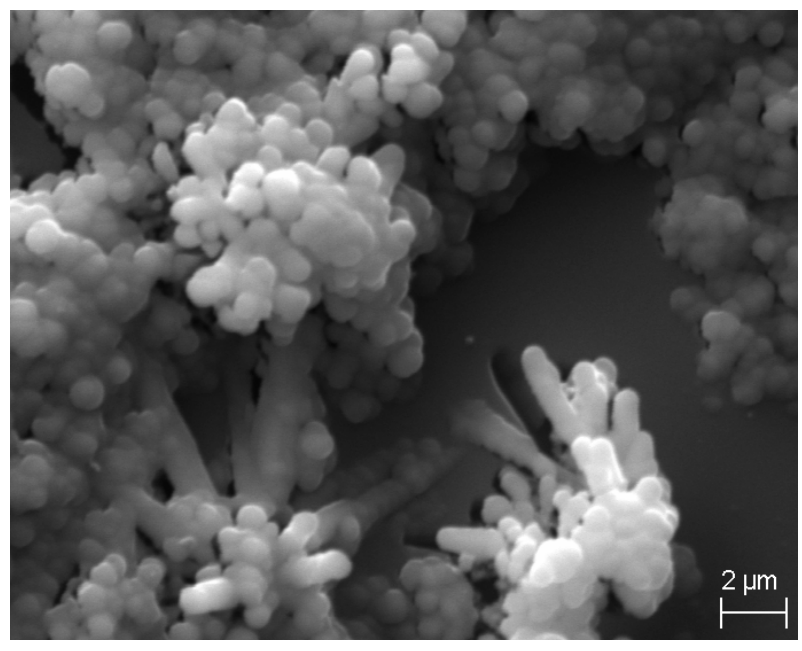

Fig. 3. SEM image showing typical particles synthesized with our evaporation technique. Both spherical and whiskers structures are evident.

obtained by coating the seed substrate with PVD vapors. Then, the steam condenses onto the seeds by covering them with a uniform layer of amorphous material. However, the needle-like shape of the seeds plays a significant role in the final morphology of the crystallized product, depending on wether the steam condensation occurs along the needle length or on the needle tip. In the former case, whisker-type structures are formed, while spherical morphologies were observed in the latter. Considering the slow-rate coating of the seeded substrate by the condensed steam, the thickness of the deposited material will uniformly cover both the spherical and the whisker structures. By changing the thickness of the evaporated layer, the granularity of the product can be controlled.

\subsection{Morphological analyses}

As explained in the introduction, the morphology of the deposited CDs is one of the most important features to be reproduced. Both the seeding and the deposition process were finely tuned to maximize the number of synthesized particles, but also to control their characteristic and distribution. After the evaporation of the starting olivine on the seeded substrate, a careful morphological study of the sample was carried out to evaluate the surface texture. Such analyses were performed with an electron microscope. The results were used as a feedback to optimize the following evaporation experiments and to optimize the technique. Representative results are displayed in Fig. 3; the scale is the same as in Fig. 2.

In this image, many clusters of spherical particles are present together with some bundles of elongated structures similar to whiskers. Both types of particles are morphologically similar to structures observed in natural CDs (Ishii et al. 2008). From this evidence, we infer that optical and spectroscopic signatures (diffused reflectivity, absorption, etc.) can be confidently considered representative of natural particles.

Unfortunately, the presence of the seed within the particle does not guarantee that the properties measured in transmission are compatible with those of natural CDs. Thus, as long as it is not possible to create CDAs without such a seed, we have to assume that our particles can only reproduce those $\mathrm{CD}$ features that do not involve the central part of the particles itself. Therefore, we assume that scattering or the interaction of CDA surfaces 


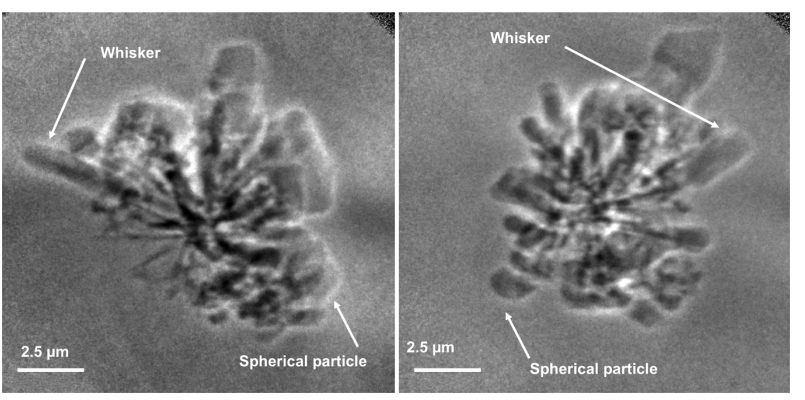

Fig. 4. X-ray images of a cluster of deposited particles. The two frames were recorded during the rotation of the particle.

with gases and chemicals are well reproduced, whereas transmission or absorption of radiation or particles is not reliable. Additionally, our synthesis is based on a slow accretion of the material onto the seed. As a consequence, spherical particles are homogeneous in size and the average dimension of the grains can be controlled.

The determination of the particle size a priori is a challenging task. Milling or plasma techniques are characterized by a highly inhomogeneous dimensional distribution of the particles, ranging from few nanometers to several micrometers in the same run. In contrast, it is possible to determine the roughness with our technique a priori by selecting the seed and controlling both deposition rate and time. The chemical composition of the synthesized particles was determined by an EDX analysis carried out on single grains and wide areas. The results agree with the values summarized in Table 1.

X-ray tomography (CT-scan) was carried out on some deposited samples in collaboration with the National Synchrotron Radiation Laboratory (NSRL) of the University of Science and Technology (Hefei, PR China). By using a binocular microscope, a cluster of particles was removed from the substrate and mounted on a remote-controlled XYZ stage at the X-ray beamline (U7A; Zhu et al. 2005; Chen et al. 2008) to perform tomography experiments. The transmission $\mathrm{X}$-ray microscope works with radiation emitted by a wiggler (magnetic field of $6 \mathrm{~T}$ ) of the NSRL source running with a current of $1 \mathrm{~A}$, monochromatized by a $\mathrm{Si}$ (111) double-crystal monochromator. The beam is focused on the sample using a capillary lens, which allows a concentration efficiency close to $90 \%$. After placing the sample, a Fresnel zone plate was used as an objective to focus radiation on the imaging scintillator. An optical system magnifies and detects the visible radiation image by a CCD camera with a field of view on the sample of $15 \times 15 \mu \mathrm{m}^{2}$. By rotating the stage, X-ray pictures were taken every $5^{\circ}$, allowing simultaneous recording of a movie of the rotation and a $3 \mathrm{D}$ tomographic reconstruction of the investigated cluster.

A small cluster was selected that included both spherical particles and whiskers. Two views are shown in Fig. 4 that were taken at different rotation angles $\left(\sim 90^{\circ}\right)$. In each image the structure of the cluster is evident. Many spherical particles surround the tip of the black filaments. Spheres like this can be easily distinguished at the top and right side of the left image, while they are more evident in the left lower part of the right image. A cylindrical protuberance (a whisker), corresponding to one of the spikes of the sea-urchin structure, can be clearly recognized in the left image. The low contrast between the particles and the background of the image is due to the low iron content area within the deposited material, while the darker part in the core of the particle cluster is due to the high Fe content of the seed. By comparing the two images, it is possible to recognize the three-dimensional structure of the cluster. A whisker and a spherical structure, whose layout changed owing to the rotation of the stage, are highlighted in red in the two images.

\section{Conclusion}

A novel technique suitable for synthesizing glassy layers from natural olivine was developed. The deposited layers have the same composition as the starting olivine. A seeding process was also implemented to produce nanoparticles and nano-structured surfaces with morphologies similar to that of CDA. This technique, based on a seeding process followed by a reactive PVD deposition of glassy silicates, was validated by FTIR, EDX, SEM, and X-ray microscopy imaging measurements. The production of amorphous magnesium and iron silicates was demonstrated, paving the way to the synthesis of many types of mineral species present in outer space. The results also demonstrate that the PVD technique we developed allows good reproducibility of the stoichiometry of the evaporated mineral species. Moreover, the morphology of the grown layers on the seeded substrate is in the nanometer range. Work is in progress to improve the seeding process so that clusters of smaller particles can be synthesized. This might be achieved by reducing the particle size and improving the dispersion of the seeds on the substrate.

The nucleation from the tips of the seeds was identified as the main growth process with respect to covering the seeds. The results show that a procedure to control the morphology of the produced CDA, based on the mutual position and orientation of the source with respect to the substrate, can be set up. More experiments on the synthesized CDA will improve the knowledge of the spectroscopic signatures in astronomical observations. In the future, we will arrange the systematic production of different types of mineral assemblies by simultaneously controlling different deposition sources during the same experiment. This will allow the control of specific element enrichment or chemical composition gradients inside the layers or particle thickness. Post-growth treatments will also be developed to simulate water metamorphosing or temperature-induced crystallization. The different mineral aggregates will be used to collect optical parameters, gas and organic molecule adsorption coefficients, and the activation energy of amorphization and crystallization processes as a function of controlled chemistry and morphologies. The final goal will be the synthesis of products with a wide range of controlled chemical or physical characteristics, that is, different crystalline and amorphous ratios, compositional modulations, differently sized particles, different surface roughness, etc. The characterization of these materials may also generate a database extremely useful for comparison in astrophysical models or for extrapolating astronomical environmental conditions.

Acknowledgements. We acknowledge the Inter-department Centre of Electronic Microscopy and Microanalysis (MEMA) of the University of Florence. Sincere thanks are due to A. Giannini and $\mathrm{He}$ Bo for the technical assistance in Florence and at the NSRL laboratory of the USTC University in Hefei and to J. R. Brucato for many fruitful discussions. We sincerely acknowledge G. Cibin for the support during experiments performed at Diamond within the proposal sp9050.

\section{References}

Apai, D., \& Lauretta, D. S. 2010, Protoplanetary Dust Astrophysical and Cosmochemical Perspective (Cambridge University Press)

Bouwman, J., de Koter, A., Dominik, C., \& Waters, L. B. F. M. 2003, A\&A, 401, 577

Cestelli Guidi, M., Piccinini, M., Marcelli, A., et al. 2005, J. Opt. Soc. Am. A, 22,2810 
Chen, Jie, Chunyan, Wu, Yangchao Tian, et al. 2008, App. Phys. Lett., 92, 233104

Colangeli, L., Henning, Th., Brucato, J. R., et al. 2003, A\&ARv, 11, 97

Crovisier, J., Leech, K., Bockelée-Morvan, D., et al. 1997, Science, 275, 1904

Crovisier, J., Encrenaz, T., Lellouch, E., et al. 1999, ISO spectroscopic observations of short-period comets, The Universe as Seen by ISO, eds. P. Cox, \& M. F. Kessler, ESA-SP, 427, 161

Deer, A., Howie, R. A., \& Zussman, J. 1997, Rock-forming minerals: singlechain silicates, Vol. 2A (London: The Geological Society)

Della Ventura, G., Marcelli, A., \& Bellatreccia, F. 2014, Rev. Min. Geochem. 78, 447

Demory, B.-O., de Wit, J., Lewis, N., et al. 2013, ApJ, 776, L25

Draine, B. T. 2011, Physics of interstellar and intergalactic medium, Princeton Ser. Astrophys. (Princeton University Press)

Duke, D. A., \& Stephens, J. D. 1964, Am. Mineralogist, 49, 1388

Gail, H. P., \& Tscharnuter, W. M. 2007, in Reactive Flows, Diffusion and

Transport (Berlin, Heidelberg: Springer), 437

Greenberg, J. M., \& Li, A. 1996, A\&A, 309, 258

Henning, T., \& Mutschke, H. 2010, J. Nanophoton, 4, 041580

Ishii, H. A., Bradley, J. P., Dai, Z. R., et al. 2008, Science, 319, 447

Jaffe, W., Meisenheimer, K., Röttgering, H. J. A., et al. 2004, Nature, 429, 47

Jing, Dapeng, Jiao, He, Brucato, J. R., et al. 2011, ApJ, 741, L9

Jing, Dapeng, Jiao, He, \& Brucato, J. R., 2012, ApJ, 756, 98
Mahan, J. E. 2000, Physical Vapor Deposition of Thin Films (Wiley-VCH), 336 Malfait, K., Waelkens, C., Waters, L. B. F., M., et al. 1998, A\&A, 332, L25

Mann, H. T. 1964, Method for the deposition of thin films by electron bombardment, Space Technology Lab Inc, Patent US3132046 A

Min, M., Waters, L. B. F. M., \& de Koter, A. 2007, A\&A, 462, 667

Mizumo, H., Markiewicz, W. J., \& Wölk, H. J. 1988, A\&A, 195, 183

Molster, F. J., Yamamura, I., Waters, L. B. F. M., et al. 1999, Nature, 401, 563

Molster, F. J., Waters, L. B. F. M., Tielens, A. G. G. M., \& Barlow, M. J. 2002, A\&A, 382, 184

Murata, K., Chihara, H., Tsuchiyama, A., Koike, C., \& Takakura, T. 2007, ApJ, 668,285

Pollack, J. B., Hollenbach, D., Beckwith, S., et al. 1994, ApJ, 421, 615

Rotundi, A., Rietmeijer, F. J. M., \& Brucato, J. R. 2000, Planet. Space Sci., 48, 371

Rotundi, A., Brucato, J. R., \& Colangeli, L. 2002, Meteor. Planet. Sci., 37, 1623

Sandford, S. A. 1996, Meteor. Planet. Sci., 31449

Verhoelst, T., Van der Zypen, N., \& Hony, S. 2009, A\&A, 98, 127

Westwood, W. D. 1989, Physical Vapor Deposition, Microelectronic Materials and Processes, NATO ASI Series, 164, 133

Whittet, D. C. B. 2002, Dust in the Galactic environment (Bristol; Philadelphia: Institute Of Physics Publishing)

Wu, Z. Y., Mottana, A., Marcelli, A., et al. 2004, Phys. Rev. B, 69, 104106

Zhu, P. P., Wang, J. Y., Yuan, Q. X., et al. 2005, Appl. Phys. Lett., 87, 264101 